\title{
DENSE CLUMPS IN NGC 2024 - PROTOSTELLAR CONDENSATIONS?
}

\author{
A. Schulz, R. Zylka, R. Güsten \\ Max-Planck-Institut für Radioastronomie, Bonn
}

It has been suggested that in NGC 2024 star forming processes are still going on, and dense cores found by $1.3 \mathrm{~mm}$ continuum observations are identified as possible protostellar condensations. To trace the molecular gas of high density, we observed the CS $(\mathrm{J}=7-6)$ line using the $30 \mathrm{~m}$ IRAM MRT for the first time at submillimetre frequencies (spat. resol. $8.5^{\prime \prime}$ ). These and complementary observations of lower $\mathrm{J} \mathrm{CS} / \mathrm{C}^{34} \mathrm{~S}$ transitions as well as the temperature probing $\mathrm{NH}_{3}(1,1)$ and $(2,2)$ non-metastable lines enable us to determine temperature and density of 3 selected cores, their kinematics and their masses. These parameters are compared with what is expected for protostellar condensations.

\section{RADIO MAPS OF THE REGIONS RCW 57 AND W 49}

\author{
N.S. Sabalisck ${ }^{1}$, Z. Abrahan ${ }^{1}$ and C.E. Tateyama ${ }^{2}$ \\ ${ }^{1}$ Instituto Astronomico e Geofisico, Sao Paulo, Brasil \\ ${ }^{2}$ Instituto de Pesquisas Espaciais, Sao Jose dos Campos, Brasil
}

We present maps of the regions RCW57 and W59 in the frequency of $22 \mathrm{GHz}$ with 4.6 angular resolution. The thermal radio sources $\mathrm{RCW} 57$ II are well resolved in the map. RCW57 I presents, at this frequency a diameter twice as large as that reported at $5 \mathrm{GHz}$, obtained with similar resolution, but coincides with the size of the source at the wavelenght of $1 \mathrm{~mm}$. In the map of the RCW57 region we detected also the supernova remnant G291.0-0.1, of plerionic type. We find a break in the spectrum of this source between 8 and $22 \mathrm{GHz}$, with the spectral index changing from -0.29 to -0.77 . This behaviour is also found in other remnats of the same type.

The region W49 includes the giant HII region W49A and the supernova remnant W49B. The structure of the map is similar to the structure found at lower frequencies with similar angular resolution. For the HII region we find an integrated flux density much larger than that of the embeded compact sources, indicating that most of the emission originates in the extended, optically thin component. The integrated flux density of the supernova remnant $\mathrm{W} 49 \mathrm{~N}$ lies well in the extrapolated spectrum of the source, with a spectral index of -0.45 between $408 \mathrm{MHz}$ and $22 \mathrm{GHz}$. 